OPEN ACCESS

Edited by:

Andrey V. Kozlov, Institute for Experimental and Clinical

Traumatology (LBG), Austria

Reviewed by:

Arthur Hosmann,

Medical University of Vienna, Austria

Evert-Jan Wils,

Franciscus Gasthuis and

Vlietland, Netherlands

*Correspondence:

Yanfei Shen

snow.shen@hotmail.com

tThese authors have contributed equally to this work and share first authorship

Specialty section:

This article was submitted to

Intensive Care Medicine and

Anesthesiology,

a section of the journal

Frontiers in Medicine

Received: 16 March 2021

Accepted: 10 August 2021

Published: 09 September 2021

Citation:

Cai G, Ru W, Xu Q, Wu J, Gong S, Yan J and Shen Y (2021) Association

Between Oxygen Partial Pressure Trajectories and Short-Term

Outcomes in Patients With

Hemorrhagic Brain Injury.

Front. Med. 8:681200.

doi: 10.3389/fmed.2021.681200

\section{Association Between Oxygen Partial Pressure Trajectories and Short-Term Outcomes in Patients With Hemorrhagic Brain Injury}

\author{
Guolong Cai ${ }^{1+}$, Weizhe $\mathrm{Ru}^{2 \dagger}$, Qianghong $\mathrm{Xu}^{1}$, Jiong $\mathrm{Wu}^{3 \dagger}$, Shijin Gong ${ }^{1}$, Jing $\mathrm{Yan}^{1 \dagger}$ and \\ Yanfei Shen ${ }^{1 *}$ \\ 1 Department of Intensive Care, Zhejiang Hospital, Hangzhou, China, ${ }^{2}$ Department of Oncology, Cixi People's Hospital, Cixi, \\ China, ${ }^{3}$ Department of Neurology, Zhejiang Hospital, Hangzhou, China
}

Objectives: Arterial hyperoxia is reportedly a risk factor for poor outcomes in patients with hemorrhagic brain injury $(\mathrm{HBI})$. However, most previous studies have only evaluated the effects of hyperoxia using static oxygen partial pressure $\left(\mathrm{PaO}_{2}\right)$ values. This study aimed to investigate the association between overall dynamic oxygenation status and $\mathrm{HBI}$ outcomes, using longitudinal $\mathrm{PaO}_{2}$ data.

Methods: Data were extracted from the Medical Information Mart for Intensive Care III database. Longitudinal $\mathrm{PaO}_{2}$ data obtained within $72 \mathrm{~h}$ of admission to an intensive care unit were analyzed, using a group-based trajectory approach. In-hospital mortality was used as the primary outcomes. Multivariable logistic models were used to explore the association between $\mathrm{PaO}_{2}$ trajectory and outcomes.

Results: Data of 2,028 patients with $\mathrm{HBI}$ were analyzed. Three $\mathrm{PaO}_{2}$ trajectory types were identified: Traj-1 (mild hyperoxia), Traj-2 (transient severe hyperoxia), and Traj-3 (persistent severe hyperoxia). The initial and maximum $\mathrm{PaO}_{2}$ of patients with Traj-2 and Traj-3 were similar and significantly higher than those of patients with Traj-1. However, $\mathrm{PaO}_{2}$ in patients with Traj-2 decreased more rapidly than in patients with Traj-3. The crude in-hospital mortality was the lowest for patients with Traj-1 and highest for patients with Traj-3 (365/1,303, 209/640, and 43/85 for Traj-1, Traj-2, and Traj-3, respectively; $p<$ 0.001 ), and the mean Glasgow Coma Scale score at discharge $\left(\right.$ GCS $\left._{\text {dis }}\right)$ was highest for patients with Traj-1 and lowest in patients with Traj-3 (13 [7-15], 11 [6-15], and 7 [3-14] for Traj-1, Traj-2, and Traj-3, respectively; $p<0.001)$. The multivariable model revealed that the risk of death was higher in patients with Traj-3 than in patients with Traj-1 (odds ratio [OR]: 3.3, 95\% confidence interval [Cl]: 1.9-5.8) but similar for patients with Traj-1 and Traj-2. Similarly, the logistic analysis indicated the worst neurological outcomes in patients with Traj-3 (OR: 3.6, 95\% Cl: 2.0-6.4, relative to Traj-1), but similar neurological outcomes for patients in Traj-1 and Traj-2.

Conclusion: Persistent, but not transient severe arterial hyperoxia, was associated with poor outcome in patients with $\mathrm{HBI}$.

Keywords: Glasgow Coma Scale, hyperoxia, hospital mortality, intracranial hemorrhage, oxygen partial pressure 


\section{INTRODUCTION}

Hemorrhagic brain injury (HBI), including traumatic/nontraumatic intracerebral hemorrhage, extra/subdural hemorrhage, and subarachnoid hemorrhage (SAH), has a distinct illness trajectory characterized by sudden, unexpected presentation and uncertain prognosis (1). Despite improvements in management, HBI still accounts for the death of $\sim 10$ million people every year and causes long-term disabilities (2,3). Many risk factors for poor outcome of various type of HBI have been identified, such as glasgow coma scale (GCS) on admission, severity of illness (4), low calcium (4) and hemoglobin levels (5), high serum lactate and blood glucose levels, and a low cholinesterase level (6). Impaired oxygenation is common among patients with $\operatorname{HBI}(7,8)$, and multiple studies have shown that prehospital hypoxia (9) or hypoxic episodes during intensive care (10) are associated with poor prognosis in patients with traumatic brain injury (TBI).

Given the evidence that hypoxemia is detrimental to patients with HBI, high-concentration oxygen therapy is frequently administered to patients with HBI to avoid hypoxemia, particularly to those who have just been admitted to the intensive care unit (ICU) and have not been adequately evaluated (11). However, this practice may cause hyperoxia, and there is accumulating evidence that hyperoxia on admission is associated with poorer outcomes in patients with $\mathrm{HBI}$, including $\mathrm{SAH}$ (12-14), TBI $(15,16)$, and stroke (17). A study (15) of 1,547 patients with TBI found that hyperoxia (based on the average arterial oxygen partial pressure $\left[\mathrm{PaO}_{2}\right]$ ) within the first $24 \mathrm{~h}$ of hospitalization was associated with worse neurological outcomes and a higher mortality, and another retrospective study (17) found that hyperoxia (based on the first $\mathrm{PaO}_{2}$ measurement) on ICU admission was associated with higher in-hospital mortality among 2,894 ventilated patients with stroke.

However, a common limitation of these studies is that only static $\mathrm{PaO}_{2}$ values were evaluated. The lack of consideration of the longitudinal dynamic $\mathrm{PaO}_{2}$ change over time may increase the risk of bias and clinical confusion. For example, it remains unclear whether high oxygen concentration should be used in HBI patients (17).

Group-based trajectory modeling has been widely used to map symptom progression and to assess heterogeneous responses to clinical interventions (18). This study addressed the limitations of previous studies by adopting a group-based trajectory approach to analyze longitudinal $\mathrm{PaO}_{2}$ data of patients admitted to the ICU with various type of HBIs. We hypothesized that different $\mathrm{PaO}_{2}$ trajectories are associated with different clinical outcomes.

\section{MATERIALS AND METHODS}

\section{Data Source}

Data were extracted from the Medical Information Mart for Intensive Care III (MIMIC III) database (19), an online database containing detailed information on more than 40,000 ICU patients from Beth Israel Deaconess Medical Center. Patient information has been anonymized for privacy. Author YS had access to this database (Certification number: 1564657) and was responsible for data extraction.

\section{Ethics Compliance}

This study was performed in accordance with the ethical standards as laid down in the 1964 Declaration of Helsinki and its later amendments. Analysis of data from this database was approved by the Institutional Review Boards of the Massachusetts Institute of Technology and Beth Israel Deaconess Medical Center. Informed consent from the patients was waived due to the retrospective nature of the study.

\section{Study Population and Stratification Method}

Preliminary screening of patients was performed by using the International Classification of Diseases, Ninth Revision (ICD 9) codes for different types of $\mathrm{HBI}$, including spontaneous or traumatic intracerebral hemorrhage, $\mathrm{SAH}$, and extra/subdural hemorrhage. The detailed causes of HBI could not be clearly distinguished due to the content of the ICD 9 codes. Therefore, all patients with any type of HBI were pooled in one cohort. The exclusion criteria were as follows: (i) age $<18$ years, (ii) no record of $\mathrm{PaO}_{2}$ levels, and (iii) records of hypoxia, defined as $\mathrm{PaO}_{2}$ value $<60 \mathrm{mmHg}$

\section{Data Extraction}

Data were collected from the MIMIC III database. These data include demographic information, comorbidity, laboratory results, transfer information, disease severity, and clinical evaluation results. The first value on ICU admission was used as the initial value for laboratory indexes and hemodynamic data and the mean/maximum laboratory indexes and hemodynamic data were calculated using the mean/maximum values during the entire ICU stay. In order to conduct the trajectory modeling, $\mathrm{PaO}_{2}$ data were extracted from the blood gas measurements obtained within $72 \mathrm{~h}$ of ICU admission, with no fixed measurement time points. For patients admitted to ICU more than once, only the first admission was included.

\section{Construction of the Group-Based Trajectory Models}

In this study, $\mathrm{PaO}_{2}$ levels within $72 \mathrm{~h}$ of $\mathrm{ICU}$ admission were used to identify patients with similar $\mathrm{PaO}_{2}$ trajectories. Mild hyperoxia was defined as patients with mild hyperoxia during the $72 \mathrm{~h}$ following admission to ICU; transient severe hyperoxia was defined as patients with high $\mathrm{PaO}_{2}$ at ICU admission that rapidly decreased to mild hyperoxia; persistent severe hyperoxia was defined as patients with persistent high $\mathrm{PaO}_{2}$ levels within $72 \mathrm{~h}$ after ICU admission. The process of constructing models was as follows: (i) the Bayesian information criterion was selected to determine the number of trajectories; (ii) the $\log$ Bayes factor $\left[2 \log _{\mathrm{e}}(\mathrm{B} 10)\right]$ was used to determine whether to use a complex model or a simple model; and (iii) the average posterior probability was calculated to evaluate the posterior probability of each individual being assigned to the corresponding $\mathrm{PaO}_{2}$ trajectory, with an acceptable value of 0.70 .

\section{Primary and Secondary Outcomes}

In-hospital mortality was used as the primary outcome, and Glasgow Coma Scale at hospital discharge $\left(\mathrm{GCS}_{\text {dis }}\right)$ was used as 
the secondary outcome. According to the $\mathrm{GCS}_{\mathrm{dis}}$, patients were classified into three categories: mild $\left(\mathrm{GCS}_{\mathrm{dis}}: 13-15\right)$, moderate $\left(\mathrm{GCS}_{\mathrm{dis}}: 9-12\right)$, and severe $\left(\mathrm{GCS}_{\mathrm{dis}}: 3-8\right)$.

\section{Missing Data Management}

Continuous data were missing at a frequency of $<5 \%$. Missing values were replaced by the mean or median value according the distribution of these variables. For binary data (such as gender), the missing value cannot be replaced by the mean value, and therefore was replaced by the default value.

\section{Statistical Analyses}

Categorical variables, which are presented as percentages, were compared using the chi-square test or Fisher's exact test. Continuous variables, which are presented as means \pm standard deviations (SD) or medians according to the data's distribution, were compared using Student's $t$-test or the Wilcoxon rank-sum test. Ordinary and ordered logistic regression methods were used to determine risk factors for in-hospital mortality and $\mathrm{GCS}_{\text {dis }}$, respectively. Multivariable logistic regression models were built as follows: first, variables for which the univariate analysis determined $p$-values of $<0.20$ were included $(20,21)$ for further multivariable analysis. Seventeen covariables were identified in this step: age, maximum Sequential Organ Failure Assessment (SOFA) score, chronic obstructive pulmonary disease (COPD), hypertension, diabetes, apnea, intubation status, white blood cell (WBC) and platelet (PLT) counts, serum calcium, potassium, sodium, blood glucose, and creatinine levels, vasopressor use, initial GCS score, and fluid balance status. Next, we used a stepwise backward elimination method to remove variables with a $p>0.05$ (COPD, diabetes, serum potassium, and fluid balance were removed in this step). Multicollinearity was tested using the variance inflation factor (VIF $\geq 5$ indicate multicollinearity)

TABLE 1 | Comparison of the characteristics of survivors and non-survivors.

\begin{tabular}{|c|c|c|c|c|}
\hline Demographics & Survivors $(n=1,411)$ & Non-survivors $(n=617)$ & Overall $(n=2,028)$ & $p$ \\
\hline Age (years) & $59.5 \pm 19.4$ & $68.4 \pm 17.5$ & $62.6 \pm 19.3$ & $<0.001$ \\
\hline Male $[n(\%)]$ & $813(57.6)$ & $334(54.1)$ & 1,147 (56.5) & 0.145 \\
\hline Weight (kg) & $78.9 \pm 19.0$ & $75.6 \pm 19.7$ & $77.9 \pm 19.3$ & $<0.001$ \\
\hline \multicolumn{5}{|l|}{ Comorbidities } \\
\hline Hypertension [n (\%)] & $584(41.3)$ & $313(50.7)$ & $897(44.2)$ & $<0.001$ \\
\hline ICU types & & & & 0.003 \\
\hline TICU [n (\%)] & $1,130(80.0)$ & $453(73.4)$ & $1,583(78.0)$ & \\
\hline $\operatorname{MICU}[n(\%)]$ & $141(10.0)$ & $93(15.0)$ & $234(11.5)$ & \\
\hline $\operatorname{CSCU}[n(\%)]$ & $104(7.3)$ & $48(7.7)$ & $152(7.4)$ & \\
\hline Time interval between hospital and ICU admission (days) & $0.27 \pm 1.8$ & $0.29 \pm 2.4$ & $0.28 \pm 2.0$ & 0.879 \\
\hline Minimum albumin level (g/dL) & $3.1 \pm 0.5$ & $2.9 \pm 0.6$ & $3.1 \pm 0.6$ & $<0.001$ \\
\hline Maximum serum creatinine (mg/dL) & $1.0 \pm 0.9$ & $1.4 \pm 1.1$ & $1.2 \pm 1.0$ & $<0.001$ \\
\hline Minimum serum sodium (mmol/L) & $135.3 \pm 4.5$ & $136.9 \pm 6.0$ & $135.8 \pm 5.1$ & $<0.001$ \\
\hline Initial glucose level (mmol/L) & $7.8 \pm 2.2$ & $8.6 \pm 3.1$ & $8.0 \pm 2.5$ & $<0.001$ \\
\hline Maximum lactate level (mmol/L) & $2.4 \pm 1.7(n=1,054)$ & $3.7 \pm 3.3(n=469)$ & $2.8 \pm 2.4(n=1,523)$ & $<0.001$ \\
\hline Initial $\mathrm{PaO}_{2}$ level (mmHg) & $211.1 \pm 121.2$ & $230.3 \pm 132.6$ & $216.9 \pm 125.0$ & $<0.001$ \\
\hline Maximum $\mathrm{PaO}_{2}$ level $(\mathrm{mmHg})$ & $256.4 \pm 119.6$ & $290.3 \pm 123.9$ & $266.7 \pm 121.9$ & $<0.001$ \\
\hline \multicolumn{5}{|l|}{ Hemodynamic data } \\
\hline Fluid balance (mL/48 h/kg) & $15.9 \pm 46.6$ & $32.6 \pm 60.9$ & $21.0 \pm 51.9$ & $<0.001$ \\
\hline Vasopressor use within 48 h [n (\%)] & $97(6.8)$ & $141(22.8)$ & $238(11.7)$ & $<0.001$ \\
\hline \multicolumn{5}{|l|}{ Clinical evaluation } \\
\hline
\end{tabular}

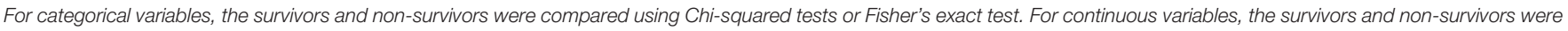
compared using Student's t-test or the Wilcoxon rank-sum test, according to the data distribution.

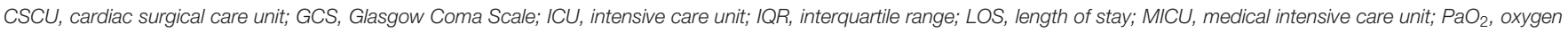
partial pressure; SOFA, Sequential Organ Failure Assessment; TICU, trauma intensive care unit; WBC, white blood cell. 
method (Serum calcium and sodium levels, and platelet count had VIFs $\geq 5$ and hence they were removed). All statistical analyses were performed using Stata 11.2 (StataCorp, College Station, TX, USA). Two-sided $p<0.05$ were considered to be statistically significant.

\section{RESULTS}

\section{Baseline Characteristics of Survivors and Non-survivors}

A total of 2,028 patients were included in this study (overall mortality rate, $30.4 \%$; mean age, 62 years; $56.5 \%$ male) and 724 patients were excluded due to lack of $\mathrm{PaO}_{2}$ data. Compared with the survivors, the non-survivors were older, and had significantly higher initial $(230.3 \pm 132.6 \mathrm{mmHg}$ and $211.1 \pm$ $121.2 \mathrm{mmHg}$, respectively, $p<0.001)$ and maximum $(290.3$ $\pm 123.9 \mathrm{mmHg}$ and $256.4 \pm 119.6 \mathrm{mmHg}$, respectively, $p<$ 0.001) $\mathrm{PaO}_{2}$ levels. The initial and maximum GCS scores and the maximum albumin level were significantly lower, while the maximum WBC count, serum creatinine and sodium levels, fluid balance, SOFA score, and proportion with vasopressor use were significantly higher among the non-survivors. A detailed comparison of the survivors and non-survivors is shown in Table 1.

\section{Partial Pressure of Oxygen-Based Trajectories}

The trajectory model selection process is shown in Supplementary Table 1. A total of 12,205 $\mathrm{PaO}_{2}$ records were extracted, and a mean of six $\mathrm{PaO}_{2}(\mathrm{SD}=5.0)$ values were recorded for each patient. The Bayesian information criterion and statistical significance determined the selection of three trajectories (Figure 1): Traj-1 (mild hyperoxia), patients with mild hyperoxia during the $72 \mathrm{~h}$ following admission to ICU; Traj-2 (transient severe hyperoxia), patients with high $\mathrm{PaO}_{2}$ at ICU admission that rapidly decreased to mild hyperoxia; and Traj-3 (persistent severe hyperoxia), patients with persistent high $\mathrm{PaO}_{2}$ levels within $72 \mathrm{~h}$ after ICU admission. The direction of Traj-2 and Traj-3 appeared similar, and compared with Traj-2, the persistence of hyperoxia in Traj-3 was primarily attributable to the higher initial $\mathrm{PaO}_{2}$ level. Furthermore, according to the logarithmic Bayes factor, each of the three $\mathrm{PaO}_{2}$ trajectories was the product of a unique quadratic function describing $\mathrm{PaO}_{2}$ as a function of time. A detailed comparison of $\mathrm{PaO}_{2}$ characteristics is shown in Table 2.

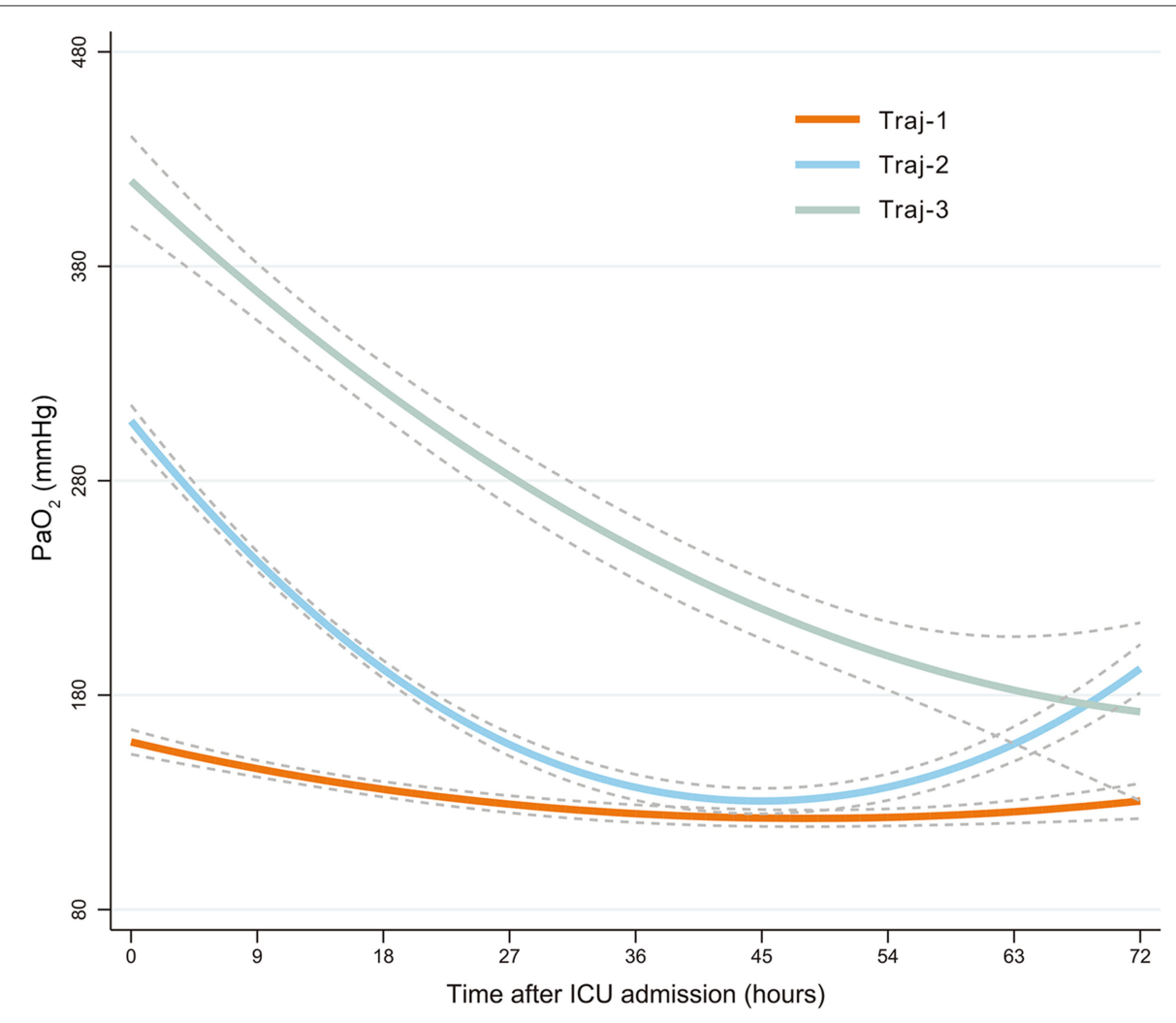

FIGURE 1 | $\mathrm{PaO}_{2}$-based trajectories of patients with HBI. Traj-1 (mild hyperoxia), patients with mild hyperoxia during the $72 \mathrm{~h}$ following admission to ICU; Traj-2 (transient severe hyperoxia), patients with high $\mathrm{PaO}_{2}$ at ICU admission that rapidly decreased to mild hyperoxia; and Traj-3 (persistent severe hyperoxia), patients with persistent high $\mathrm{PaO}_{2}$ levels within $72 \mathrm{~h}$ after ICU admission. Group-based trajectory modeling was used to establish these $\mathrm{PaO}_{2}$ trajectories. PaO $\mathrm{O}_{2}$, oxygen partial pressure; HBI, hemorrhagic brain injury; ICU, intensive care unit. 
TABLE 2 | Partial pressure of oxygen indexes according to the partial pressure of oxygen trajectory.

\begin{tabular}{|c|c|c|c|c|}
\hline & Traj-1 $(n=1,303)$ & Traj-2 $(n=640)$ & Traj-3 $(n=85)$ & $p$ \\
\hline $\mathrm{PaO}_{2}$ on admission $(\mathrm{mmHg})$ & $149.4 \pm 68.1$ & $332.6 \pm 106.1^{\star \star}$ & $396.7 \pm 129.0^{\star \star, \# \#}$ & $<0.001$ \\
\hline Maximum $\mathrm{PaO}_{2}(\mathrm{mmHg})$ & $188.1 \pm 75.8$ & $367.6 \pm 90.1^{\star \star}$ & $455.8 \pm 88.1^{\star \star, \# \#}$ & $<0.001$ \\
\hline Minimum $\mathrm{PaO}_{2}(\mathrm{mmHg})$ & $100.0 \pm 39.7$ & $141.4 \pm 66.8^{\star \star}$ & $258.3 \pm 157.8^{\star \star, \# \#}$ & $<0.001$ \\
\hline Mean $\mathrm{PaO}_{2}(\mathrm{mmHg})$ & $132.8 \pm 36.1$ & $221.3 \pm 49.8^{\star \star}$ & $346.1 \pm 110.2^{\star \star, \# \#}$ & $<0.001$ \\
\hline Time to maximum $\mathrm{PaO}_{2}(\mathrm{~h})$ & $18.0 \pm 19.6$ & $8.2 \pm 14.0^{\star \star}$ & $17.4 \pm 14.0^{\# \#}$ & $<0.001$ \\
\hline $\mathrm{PaO}_{2}>150$ mmHg, $n(\%)$ & $2,220 / 7,610(29.1)$ & $2,818 / 4,167(67.6)^{\star \star}$ & $321 / 428(86.7)^{\star \star}$ & $<0.001$ \\
\hline $\mathrm{PaO}_{2}>200$ mmHg, $n$ (\%) & 699/7,610 (9.2) & $1,543 / 4,167(37.0)^{\star *}$ & $288 / 428(67.3)^{\star \star}$ & $<0.001$ \\
\hline $\mathrm{PaO}_{2}>250$ mmHg, $n(\%)$ & $271 / 7,610(3.6)$ & $924 / 4,167(22.2)^{\star \star}$ & $223 / 428(52.1)^{\star \star}$ & $<0.001$ \\
\hline \multicolumn{5}{|l|}{ Daily maximum $\mathrm{PaO}_{2}$} \\
\hline $\mathrm{PaO}_{2}$ on Day $1(\mathrm{mmHg})$ & $181.6 \pm 73.4$ & $363.5 \pm 94.3^{\star \star}$ & $470.1 \pm 95.8^{\star \star, \# \#}$ & $<0.001$ \\
\hline $\mathrm{PaO}_{2}$ on Day $2(\mathrm{mmHg})$ & $142.2 \pm 60.0$ & $180.3 \pm 68.8^{\star \star}$ & $334.0 \pm 111.9^{\star \star, \# \#}$ & $<0.001$ \\
\hline $\mathrm{PaO}_{2}$ on Day $3(\mathrm{mmHg})$ & $136.4 \pm 48.8$ & $176.3 \pm 71.7^{\star \star}$ & $231.7 \pm 117.7^{\star \star, \# \#}$ & $<0.001$ \\
\hline \multicolumn{5}{|l|}{ Daily mean $\mathrm{PaO}_{2}$} \\
\hline $\mathrm{PaO}_{2}$ on Day $1(\mathrm{mmHg})$ & $140.1 \pm 39.7$ & $251.2 \pm 53.3^{\star \star}$ & $405.0 \pm 103.6^{\star \star, \# \#}$ & $<0.001$ \\
\hline $\mathrm{PaO}_{2}$ on Day $2(\mathrm{mmHg})$ & $122.1 \pm 40.9$ & $155.1 \pm 44.6^{\star \star}$ & $261.3 \pm 81.2^{\star \star, \# \#}$ & $<0.001$ \\
\hline $\mathrm{PaO}_{2}$ on Day $3(\mathrm{mmHg})$ & $117.5 \pm 32.1$ & $149.4 \pm 44.4^{\star \star}$ & $187.0 \pm 62.7^{\star \star, \# \#}$ & $<0.001$ \\
\hline Number of $\mathrm{PaO}_{2}$ measurements & $5.8 \pm 5.2$ & $6.5 \pm 4.5^{\star}$ & $5.0 \pm 4.3^{\#}$ & 0.004 \\
\hline
\end{tabular}

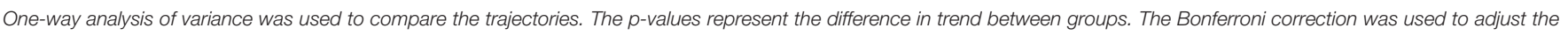

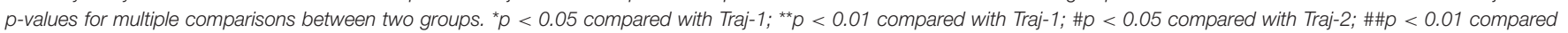
with Traj-2.

$\mathrm{PaO}_{2}$, partial pressure of oxygen, Traj-1, mild hyperoxia; Traj-2, transient severe hyperoxia; Traj-3, persistent severe hyperoxia.

\section{Mechanical Parameters According to the Partial Pressure of Oxygen Trajectory}

The mechanical parameters according to $\mathrm{PaO}_{2}$ trajectory, including fraction of inspired oxygen $\left(\mathrm{FiO}_{2}\right)$, minute ventilation, tidal volume, positive end expiratory pressure (PEEP), and respiratory rate, are shown in Table 3. The initial $\mathrm{FiO}_{2}$ (within $3 \mathrm{~h}$ after ICU admission) was comparable between Traj-2 and Traj-3, and was significantly higher in the Traj- 2 and Traj- 3 groups than that in the Traj-1 group $(79 \pm 24,81 \pm 26$, and $65 \pm 24$ for Traj2 , Traj-3, and Traj-1 groups, respectively, $p<0.001)$. However, both the mean and maximum $\mathrm{FiO}_{2}$ were significantly higher in the Traj-3 group than those in the Traj-1 and Traj-2 groups. Compared with Traj-1, the initial/mean minute ventilation, the maximum tidal volume, the initial/mean/maximum PEEP, and the initial and mean respiratory rate were significantly lower in Traj-2, and the initial/mean/maximum minute ventilation, maximum tidal volume, initial/mean/maximum PEEP, and the initial and mean respiratory rate were significantly lower in Traj-3. There were no significant differences between Traj-2 and Traj-3 in the minute ventilation, tidal volume, PEEP, or the respiratory rate.

\section{Clinical Outcomes According to the Partial Pressure of Oxygen Trajectory}

The patients with Traj-1 had the lowest in-hospital mortality and best neurological outcomes (Figure 2 and Supplementary Table 2). Although the initial and the maximum $\mathrm{PaO}_{2}$ level were similar in Traj-2 and Traj-3, the mortality was significantly higher, and the GCS dis level was significantly lower in Traj-3 (in-hospital mortality: 32.7 and 50.6\%; GCS $_{\text {dis }}$ :
11 and 7 for Traj-2 and Traj-3, respectively; both $p<0.001$ ). Detailed comparisons of the three trajectories are provided in Supplementary Table 2. The result of univariate analysis is provided in Supplementary Table 3. In the multivariable logistic regression model (Table 4), relative to Traj-1 group, the risk of mortality was significantly higher in the Traj- 3 group (odds ratio [OR]: $3.3,95 \%$ confidence interval [CI]: 1.9-5.8) but not in the Traj-2 group (OR: 1.2, 95\% CI: 0.9-1.5). Similarly, the logistic regression analysis (Table 4) revealed that, relative to the Traj-1 group, the neurological outcomes were significantly worse in the Traj-3 group (OR: 3.6, 95\% CI: 2.0-6.4) but not in the Traj-2 group (OR: 1.0, 95\% CI: 0.8-1.3). In addition, age $>65$ years, intubation, hypertension, diabetes, SOFA score, high initial blood glucose, and maximum WBC count were significant risk factors for in-hospital mortality and a poor neurological outcome, while apnea and high GCS on admission were associated with lower in-hospital mortality and a better neurological outcome.

\section{DISCUSSION}

The current study has two major findings. First, based on the longitudinal $\mathrm{PaO}_{2}$ data, we identified three $\mathrm{PaO}_{2}$ trajectories in patients with $\mathrm{HBI}$, with significantly different clinical outcomes. Second, persistent hyperoxia was associated with poor outcomes in patients with HBI, while transient hyperoxia was not.

Impaired pulmonary oxygenation is common among patients with $\operatorname{HBI}(9,22,23)$. However, as there is evidence that cerebral ischemia can be alleviated by increasing $\mathrm{PaO}_{2}$ levels to ensure adequate oxygen delivery to the brain (12), high-concentration oxygen therapy is commonly applied to brain-injured patients, 
TABLE 3 | Respiratory parameters according to the partial pressure of oxygen trajectory.

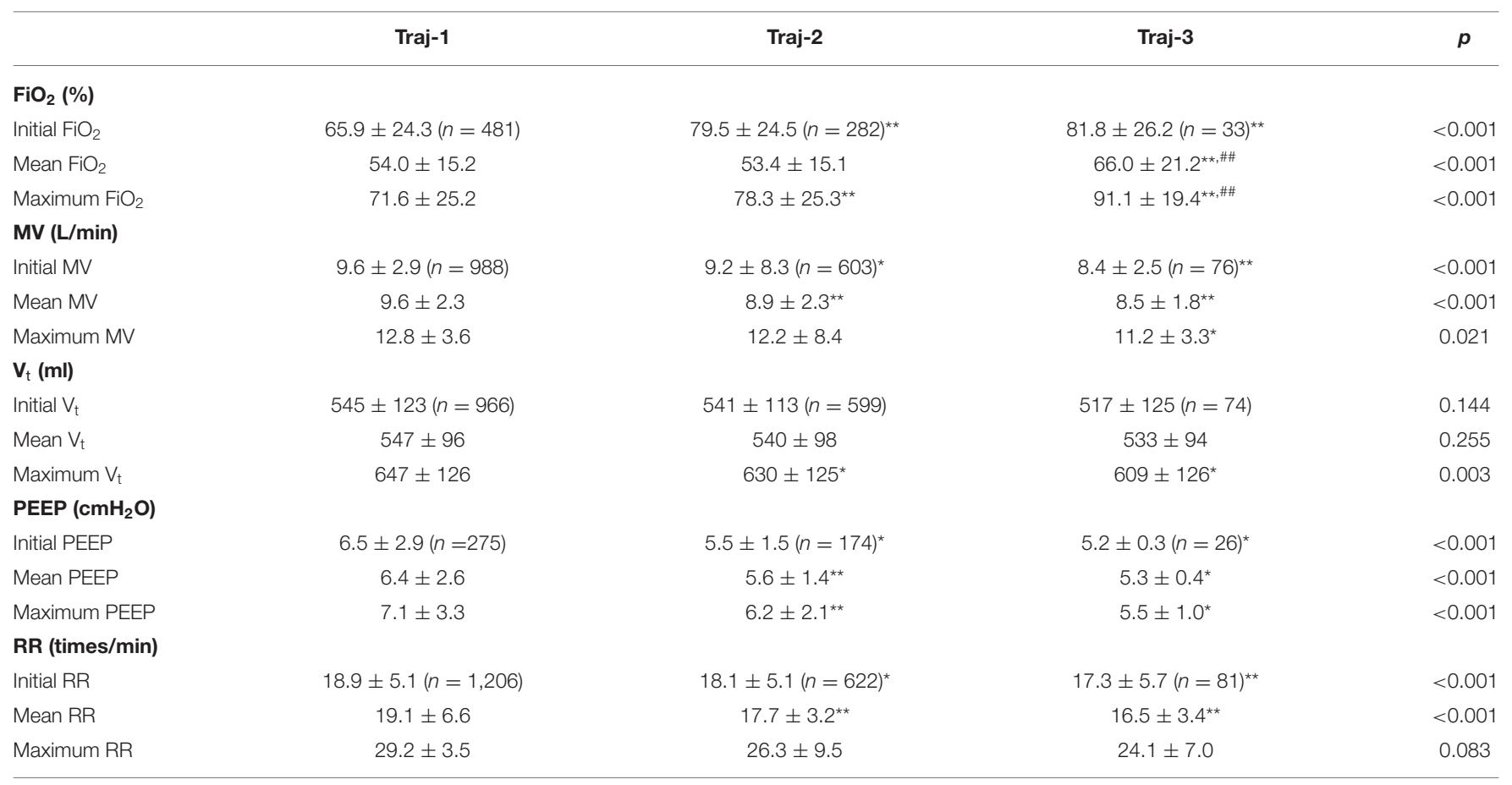

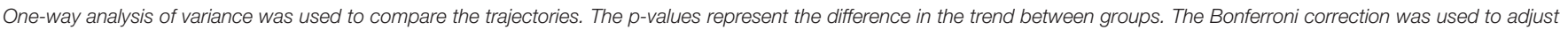

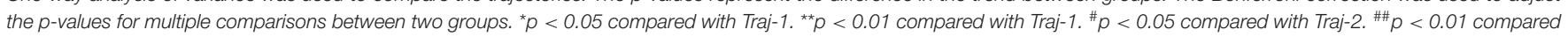
with Traj-2.

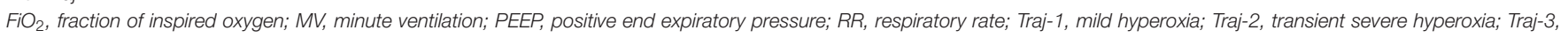
persistent severe hyperoxia; Vt, tidal volume.

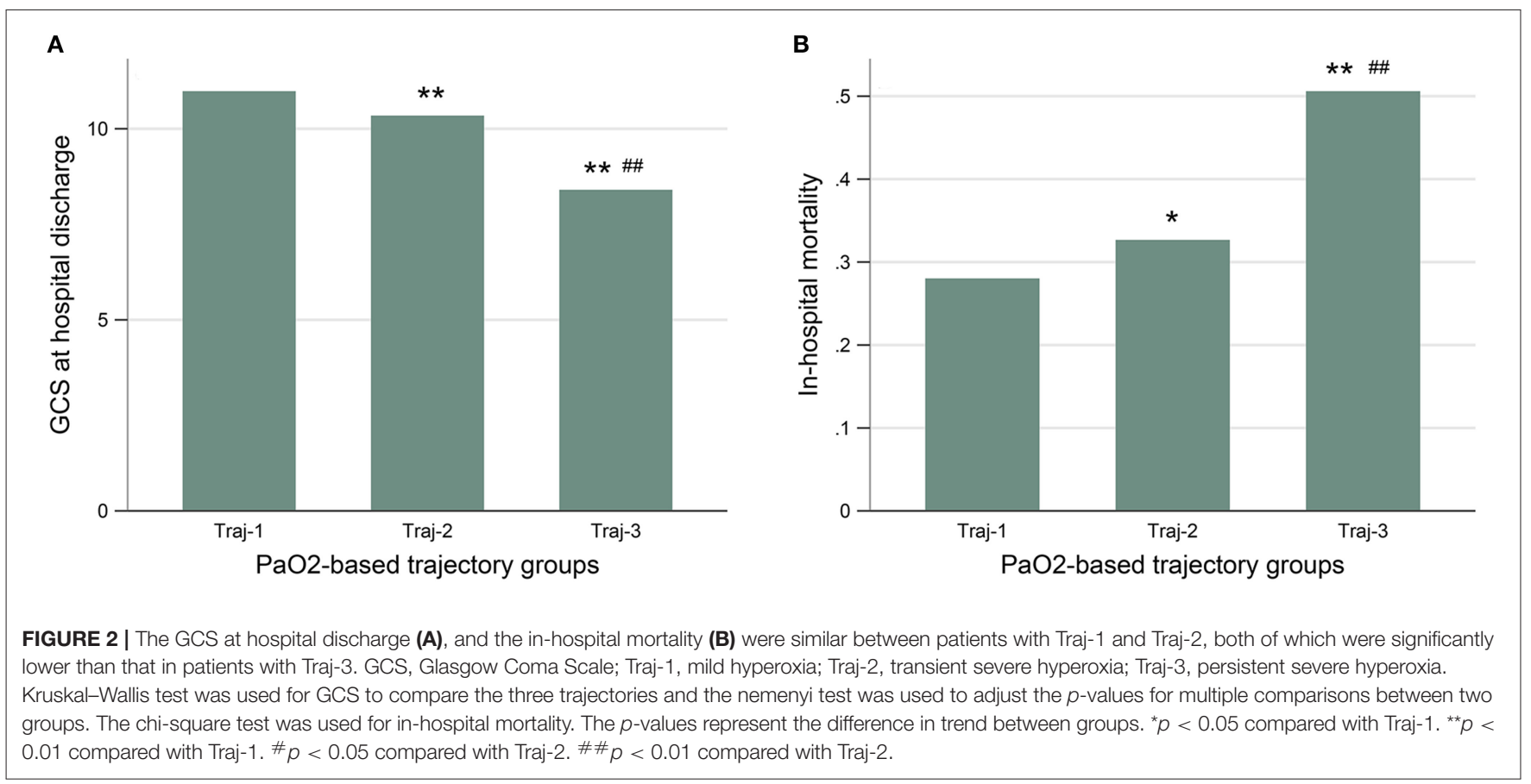


TABLE 4 | Associations between the partial pressure of oxygen trajectory and prognosis in multivariable logistic regression models.

\begin{tabular}{|c|c|c|c|c|c|}
\hline \multicolumn{3}{|c|}{ In-hospital mortality (Model A) } & \multicolumn{3}{|c|}{ Poor neurological outcome (Model B) } \\
\hline Variables & Adjusted OR (95\% Cl) & $\boldsymbol{P}$ & Variables & Adjusted OR (95\% Cl) & $p$ \\
\hline Traj-1 & Reference & - & Traj-1 & Reference & - \\
\hline Traj-2 & $1.2(0.9-1.5)$ & 0.101 & Traj-2 & $1.0(0.8-1.3)$ & 0.532 \\
\hline Traj-3 & $3.3(1.9-5.8)$ & $<0.001$ & Traj-3 & $3.6(2.0-6.4)$ & $<0.001$ \\
\hline Age > 65 (years) & $3.2(2.5-4.1)$ & $<0.001$ & Age > 65 (years) & $2.7(2.1-3.4)$ & $<0.001$ \\
\hline Intubated & $1.4(1.1-1.8)$ & 0.006 & Intubated & $1.5(1.1-1.9)$ & 0.001 \\
\hline Hypertension & $1.5(1.2-1.9)$ & 0.001 & Hypertension & $1.3(1.1-1.7)$ & 0.009 \\
\hline Initial glucose level & 1.006 (1.003-1.008) & $<0.001$ & Initial glucose level & 1.008 (1.040-1.009) & $<0.001$ \\
\hline Apnea & $0.2(0.1-0.5)$ & 0.001 & Apnea & $0.3(0.1-0.7)$ & 0.009 \\
\hline Maximum SOFA score & $1.2(1.1-1.2)$ & $<0.001$ & Maximum SOFA score & $1.2(1.1-1.3)$ & $<0.001$ \\
\hline Maximum WBC count & $1.02(1.00-1.03)$ & 0.008 & Maximum WBC count & $1.02(1.00-1.03)$ & 0.010 \\
\hline Maximum creatinine level & $1.2(1.1-1.3)$ & 0.001 & Maximum creatinine level & $1.1(1.0-1.2)$ & 0.021 \\
\hline GCS on admission & $0.8(0.8-0.8)$ & $<0.001$ & GCS on admission & $0.8(0.7-0.8)$ & $<0.001$ \\
\hline
\end{tabular}

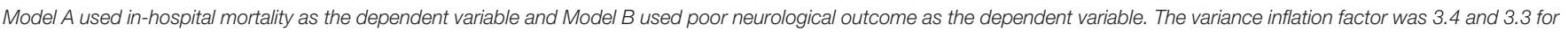
Models $A$ and $B$, respectively.

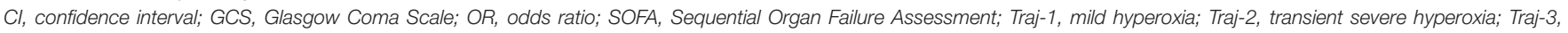
persistent severe hyperoxia; WBC, white blood cell.

especially in those who have not been adequately evaluated (11), to prevent hypoxia $(24,25)$. One recent guideline (11) on oxygen therapy suggests that initial oxygen therapy with 15 $\mathrm{L} / \mathrm{min}$ with a reservoir mask should be provided to patients before oximetry data are obtained. However, these strategies often lead to transient hyperoxia, and the role of hyperoxia in the neurocritical care setting remains controversial. Most previous studies $(13-16,26)$ have found that hyperoxia was associated with poorer neurological outcomes and increased mortality. For example, in a retrospective analysis of 3,420 patients with TBI, Davis et al. (16) found that both hypoxia and hyperoxia on hospital arrival in severe TBI were associated with increased mortality and a decrease in neurological function, compared with normoxia. Another retrospective study (26) in TBI also found that an elevated first $\mathrm{PaO}_{2}$ value during the first $24 \mathrm{~h}$ in ICU was associated with higher in-hospital mortality. In addition, Rincon et al. (17) reported that in patients with ventilated stroke (both ischemic and hemorrhagic), $\mathrm{PaO}_{2} \geq 300 \mathrm{mmHg}$ was independently associated with an increased risk of in-hospital death compared with patients with either normoxia or hypoxia. Similar findings have been reported in patients with $\mathrm{SAH}(13,14)$. However, other studies with different findings were also reported. In a national multicenter cohort study (27) of 3,033 adult patients with spontaneous ICH, Fallenius et al. (27) found no significant relation between the $\mathrm{PaO}_{2}$ levels (hyperoxia: $>150 \mathrm{mmHg}$ ) and long-term mortality. Another study (28) found that early moderate hyperoxia $(>150 \mathrm{mmHg}$ ) did not increase or decrease the risk of a poor outcome in mechanically ventilated patients with SAH.

Different definitions for hyperoxia may be one important reason for these inconsistent findings. Previous studies that investigated the effect of hyperoxia on patients with different types of HBI used different definitions of hypoxia $\left(\mathrm{PaO}_{2} \geq\right.$
$150,>200,>300$, and $>487 \mathrm{mmHg}$ ), based on single $\mathrm{PaO}_{2}$ values measured at different time points, such as the first $\mathrm{PaO}_{2}$ within the first $24 \mathrm{~h}$ after admission, $\mathrm{PaO}_{2}$ value from the Acute Physiology and Chronic Health Enquiry score on the first day, and mean $\mathrm{PaO}_{2}$ (Supplementary Table 4). In this study, we explored the $\mathrm{PaO}_{2}$ trajectories in real clinical situations. We noted that the overall $\mathrm{PaO}_{2}$ was high in the whole cohort of $\mathrm{HBI}$. In clinical practice, physicians may be mostly concerned about avoiding hypoxia and give additional oxygen as a precaution, as the deleterious effects of hypoxia are well-known $(29,30)$. Therefore, supplementary oxygen is routinely applied to brain-injured patients, even in those with adequate oxygen saturation, which may lead to transient or persistent hyperoxia.

In addition, another limitation of previous studies on the associations between hyperoxia and HBI outcome is that hyperoxia was mainly defined using a single $\mathrm{PaO}_{2}$ value, which may not have reflected the patients' daily $\mathrm{PaO}_{2}$ status. In studies that based the definition of hyperoxia on a single measurement value, both the transient and persistent hyperoxia were classified as hyperoxia. To solve this limitation, several strategies have been proposed to reflect the overall $\mathrm{PaO}_{2}$ status, such as average $\mathrm{PaO}_{2}$ (15), TWA- $\mathrm{PaO}_{2}(13,28)$, and oxygen burden (14). For example, in a single-center retrospective study with 197 patients with $\mathrm{SAH}$, Fukuda et al. (13) reported that a high TWA- $\mathrm{PaO}_{2}$ during the first $24 \mathrm{~h}$ was associated with delayed cerebral ischemia and a poor prognosis. Another study (14) of 252 patients with $\mathrm{SAH}$ also found that a high oxygen burden $(\geq 173 \mathrm{mmHg})$ was significantly associated with delayed cerebral ischemia. Although these analytic approaches consider the overall oxygenation status, they still have some notable limitations, such as: (i) hyperoxia and hypoxia status may have been normalized as a result of being averaged (15); (ii) only static $\mathrm{PaO}_{2}$ values were used in 
the analysis; and (iii) complex calculations (TWA- $\mathrm{PaO}_{2}$, oxygen burden) were used, and yet, these studies did not assess the relative effects of prolonged and transient hyperoxia in patients with HBI.

In the current study, three $\mathrm{PaO}_{2}$ trajectories were identified based on longitudinal $\mathrm{PaO}_{2}$ data, using a group-based trajectory modeling approach (18). The HBI patients with mild hyperoxia had the highest survival rate and the most favorable neurological outcomes. This finding is consistent with previous reports showing that a moderately high $\mathrm{PaO}_{2}$ is associated with favorable outcomes in patients with TBI $(16,26)$. There were some notable differences between the Traj- 2 and Traj-3 groups. Although the $\mathrm{PaO}_{2}$ on ICU admission and the maximum value were similar in the Traj- 2 and Traj- 3 groups, the outcome of the two trajectories differed considerably. Compared with the Traj-2 group, the Traj3 group had a more gradual decline in the $\mathrm{PaO}_{2}$ level, and was associated with a lower GCS score and a lower survival rate. This suggests that in patients with $\mathrm{HBI}$, transient hyperoxia is not detrimental and that only persistent hyperoxia is associated with poor outcomes.

The reason behind the different $\mathrm{PaO}_{2}$ status within these trajectories remains unclear. We noted that the initial $\mathrm{FiO}_{2}$ was comparable between the Traj- 2 and Traj- 3 groups, and was significantly higher than that in the Traj-1 group. This is consistent with above finding that the initial $\mathrm{PaO}_{2}$ was significantly higher in the Traj-2 and Traj-3 groups than that in the Traj-1 group. In addition, both the mean and maximum $\mathrm{FiO}_{2}$ were significantly higher in the Traj-3 group than those in Traj1 and Traj-2 groups. This also corresponds to the subsequent $\mathrm{PaO}_{2}$ changes within these trajectories. In addition, we found that other mechanical parameters including minute ventilation, tidal volume, and PEEP were comparable within the three trajectories; therefore, hyperoxia was mainly caused by high FiO2 levels. Our data suggest that persistent hyperoxia, which is associated with a poor outcome, may be avoided by proper adjustment of the $\mathrm{FiO}_{2}$ levels.

The mechanism between hyperoxia and poor prognosis cannot be inferred from this study. Under experimental conditions (31), hyperoxia can decrease heart rate and cardiac output. Studies in healthy subjects have found that hyperoxia is associated with decreased cerebral blood flow (32). Therefore, in addition to increased radical oxygen species (33), hyperoxia could cause decreased oxygen delivery to the brain-injured region if the increase in arterial $\mathrm{O}_{2}$ level during hyperoxia is counterbalanced by a decrease in cardiac output and cerebral flow. More studies are needed to explore the potential mechanism.

We identified several factors associated with prognosis in the multivariable logistic regression analysis, including age $>$ 65 years, intubation, hypertension, diabetes, apnea, SOFA score, glucose level, WBC count, and GCS on admission. These results are in line with previous findings that age (34), intubation (35), GCS on admission, severity of illness (4), hypertension, diabetes (36), apnea, high blood glucose levels (6), and WBC count (37) are significant predictors of the outcomes in patients with various $\mathrm{HBI}$.

\section{Strengths and Limitations}

To the best of our knowledge, this study is the first to evaluate the association between the dynamic $\mathrm{PaO}_{2}$ changes and the clinical outcomes in patients with $\mathrm{HBI}$ using a $\mathrm{PaO}_{2}$-based trajectory model. The study has some limitations. First, only shortterm outcomes were evaluated, and the relationship between transient/prolonged exposure to $\mathrm{PaO}_{2}$ and long-term outcomes, such as the 3-month GOS and the modified Rankin score, were not investigated due to a lack of data. Second, the old ICD 9 codes were used to identify patients with HBI in the current study. However, the bleeding type of these patients cannot be inferred due to the content of the ICD 9 codes. Therefore, patients with different types of HBI were pooled in one cohort. Third, although we considered the data on all the available confounders, there may be some residual confounding because some unmeasured potential confounders (e.g., surgery details and intracranial pressure) were not included in the multivariable logistic regression models. In addition, other factors such as GCS, age, intubation status also had significant associations with the prognosis. These findings should be interpreted with caution. Fourth, the data were collected from 2001 to 2012, which hinders the interpretation of these results as the findings may have changed over time due to changes in clinical management. However, as hyperoxia is a clinical phenomenon rather than a clinical treatment strategy, changes in treatment strategies since 2012 are not likely to have altered the effect of hyperoxia on the prognosis of patients with HBIs. Fifth, the time period between onset of the HBI and hospital admission may have a significant association with the prognosis of these patients. However, this information was not available in the study database. In addition, $\mathrm{PaCO}_{2}$ is an important index reflecting respiratory status and may influence intracranial physiology. However, due to the lack of data, the potential confounding effects of $\mathrm{PaCO}_{2}$ were not investigated. Finally, the number of patients in the Traj-3 group was small, and a validation cohort was consequently not isolated from the whole cohort.

\section{CONCLUSIONS}

We identified three $\mathrm{PaO}_{2}$ trajectories among patients with HBI who were admitted to the ICU. Patients with transient hyperoxia and normoxia had similar clinical outcomes, while those with persistent hyperoxia had worse neurological outcomes and higher mortality rates than did the patients from the other two groups. Further studies are needed to validate our findings and to determine whether there is a causal relationship between the $\mathrm{PaO}_{2}$ trajectory and the outcome in patients with HBI. Also, the association between the $\mathrm{PaO}_{2}$ trajectory and intracranial physiology and long-term outcomes needs to be further investigated.

\section{DATA AVAILABILITY STATEMENT}

The raw data supporting the conclusions of this article will be made available by the authors, without undue reservation. 


\section{ETHICS STATEMENT}

The studies involving human participants were reviewed and approved by Institutional Review Boards of the Massachusetts Institute of Technology and Beth Israel Deaconess Medical Center, USA. Written informed consent for participation was not required for this study in accordance with the national legislation and the institutional requirements.

\section{AUTHOR CONTRIBUTIONS}

GC and JY designed the study. QX and JW analyzed the data and prepared the figures. YS and SG collected and verified all data. WR and YS wrote the draft of the manuscript. JW made critical revisions. All authors gave final approval of the version to be published and agreed to be accountable

\section{REFERENCES}

1. Creutzfeldt CJ, Longstreth WT, Holloway RG. Predicting decline and survival in severe acute brain injury: the fourth trajectory. BMJ. (2015) 351:h3904. doi: 10.1136/bmj.h3904

2. Sakr Y, Rubatto Birri PN, Kotfis K, Nanchal R, Shah B, Kluge S, et al. Higher fluid balance increases the risk of death from sepsis: results from a Large International Audit. Crit Care Med. (2017) 45:38694. doi: 10.1097/CCM.0000000000002189

3. Silva JM Jr, de Oliveira AM, Nogueira FA, Vianna PM, Pereira Filho MC, Dias LF, et al. The effect of excess fluid balance on the mortality rate of surgical patients: a multicenter prospective study. Crit Care. (2013) 17:R288. doi: 10.1186/cc12673

4. Epstein D, Freund Y, Marcusohn E, Diab T, Klein E, Raz A, et al. Association between ionized calcium level and neurological outcome in endovascularly treated patients with spontaneous subarachnoid hemorrhage: a retrospective Cohort Study. Neurocrit Care. (2021). doi: 10.1007/s12028-02101214-3

5. Acosta JN, Leasure AC, Kuohn LR, Both CP, Petersen NH, Sansing LH, et al. Admission hemoglobin levels are associated with functional outcome in spontaneous intracerebral hemorrhage. Cri Care Med. (2021) 49:82837. doi: 10.1097/CCM.0000000000004891

6. Bender M, Naumann T, Uhl E, Stein M. Early serum biomarkers for intensive care unit treatment within the first 24 hours in patients with intracerebral hemorrhage. J Neurol Surg A Cent Eur Neurosurg. (2021) 82:13846. doi: 10.1055/s-0040-1716516

7. Junttila E, Ala-Kokko T, Ohtonen P, Vaarala A, Karttunen A, Vuolteenaho $\mathrm{O}$, et al. Neurogenic pulmonary edema in patients with nontraumatic intracerebral hemorrhage: predictors and association with outcome. Anesth Analg. (2013) 116:855-61. doi: 10.1213/ANE.0b013e31828 $11 \mathrm{cc} 7$

8. Baumann A, Audibert G, McDonnell J, Mertes PM. Neurogenic pulmonary edema. Acta Anaesthesiol Scand. (2007) 51:44755. doi: 10.1111/j.1399-6576.2007.01276.x

9. Chi JH, Knudson MM, Vassar MJ, McCarthy MC, Shapiro MB, Mallet $\mathrm{S}$, et al. Prehospital hypoxia affects outcome in patients with traumatic brain injury: a prospective multicenter study. J Trauma. (2006) 61:113441. doi: 10.1097/01.ta.0000196644.64653.d8

10. Chang JJ, Youn TS, Benson D, Mattick H, Andrade N, Harper CR, et al. Physiologic and functional outcome correlates of brain tissue hypoxia in traumatic brain injury. Crit Care Med. (2009) 37:28390. doi: 10.1097/CCM.0b013e318192fbd7

11. O'Driscoll BR, Howard LS, Earis J, Mak V, British Thoracic Society Emergency Oxygen Guideline G, Group BTSEOGD. BTS guideline for oxygen use in adults in healthcare and emergency settings. Thorax. (2017) 72:ii1ii90. doi: 10.1136/thoraxjnl-2016-209729 for all aspects of the work in ensuring that questions related to the accuracy or integrity of any part of the work are answered.

\section{FUNDING}

This work was supported by a grant from the Zhejiang Medical Association (No. 2019ZYC-A73) to YS. GC also received a funding from Zhejiang Medicine and Health Science and Technology Project (No. WKJ-ZJ-2001).

\section{SUPPLEMENTARY MATERIAL}

The Supplementary Material for this article can be found online at: https://www.frontiersin.org/articles/10.3389/fmed. 2021.681200/full\#supplementary-material

12. Bohman LE, Pisapia JM, Sanborn MR, Frangos S, Lin E, Kumar M, et al. Response of brain oxygen to therapy correlates with long-term outcome after subarachnoid hemorrhage. Neurocrit Care. (2013) 19:3208. doi: 10.1007/s12028-013-9890-6

13. Fukuda S, Koga Y, Fujita M, Suehiro E, Kaneda K, Oda Y, et al. Hyperoxemia during the hyperacute phase of aneurysmal subarachnoid hemorrhage is associated with delayed cerebral ischemia and poor outcome: a retrospective observational study. J Neurosurg. (2019) 15:18. doi: 10.3171/2019.9.JNS19781

14. Jeon SB, Choi HA, Badjatia N, Schmidt JM, Lantigua H, Claassen J, et al. Hyperoxia may be related to delayed cerebral ischemia and poor outcome after subarachnoid haemorrhage. J Neurol Neurosurg Psychiatry. (2014) 85:13017. doi: 10.1136/jnnp-2013-307314

15. Brenner M, Stein D, Hu P, Kufera J, Wooford M, Scalea T. Association between early hyperoxia and worse outcomes after traumatic brain injury. Arch Surg. (2012) 147:1042-6. doi: 10.1001/archsurg.2012.1560

16. Davis DP, Meade W, Sise MJ, Kennedy F, Simon F, Tominaga G, et al. Both hypoxemia and extreme hyperoxemia may be detrimental in patients with severe traumatic brain injury. J Neurotrauma. (2009) 26:221723. doi: $10.1089 /$ neu.2009.0940

17. Rincon F, Kang J, Maltenfort M, Vibbert M, Urtecho J, Athar $\mathrm{MK}$, et al. Association between hyperoxia and mortality after stroke: a multicenter cohort study. Crit Care Med. (2014) 42:387-96. doi: 10.1097/CCM.0b013e3182a27732

18. Nagin DS, Odgers CL. Group-based trajectory modeling in clinical research. Annu Rev Clin Psychol. (2010) 6:10938. doi: 10.1146/annurev.clinpsy.121208.131413

19. Johnson AE, Pollard TJ, Shen L, Lehman LW, Feng M, Ghassemi M, et al. MIMIC-III, a freely accessible critical care database. Sci Data. (2016) 3:160035. doi: 10.1038/sdata.2016.35

20. Mickey RM, Greenland S. The impact of confounder selection criteria on effect estimation. Am J Epidemiol. (1989) 129:12537. doi: 10.1093/oxfordjournals.aje.a115101

21. Zhang Z, Lu B, Ni H, Sheng X, Jin N. Prediction of pulmonary edema by plasma protein levels in patients with sepsis. J Crit Care. (2012) 27:6239. doi: 10.1016/j.jcrc.2012.08.007

22. Vespa PM, Bleck TP. Neurogenic pulmonary edema and other mechanisms of impaired oxygenation after aneurysmal subarachnoid hemorrhage. Neurocrit Care. (2004) 1:157-70. doi: 10.1385/NCC:1:2:157

23. Chesnut RM, Marshall LF, Klauber MR, Blunt BA, Baldwin N, Eisenberg HM, et al. The role of secondary brain injury in determining outcome from severe head injury. J Trauma. (1993) 34:216-22. doi: 10.1097/00005373-199302000-00006

24. Brain Trauma Foundation, American Association of Neurological Surgeons, Congress of Neurological Surgeons, Joint Section on Neurotrauma and Critical Care, AANS/CNS, Bratton SL, Chestnut RM, et al. Guidelines 
for the management of severe traumatic brain injury. VI. Indications for intracranial pressure monitoring. J Neurotrauma. (2007) 24(Suppl. 1):S37S44. doi: 10.1089/neu.2007.9990

25. Rajajee V, Riggs B, Seder DB. Emergency neurological life support: airway, ventilation, and sedation. Neurocrit Care. (2017) 27:4-28. doi: 10.1007/s12028-017-0451-2

26. Rincon F, Kang J, Vibbert M, Urtecho J, Athar MK, Jallo J. Significance of arterial hyperoxia and relationship with case fatality in traumatic brain injury: a multicentre cohort study. J Neurol Neurosurg Psychiatry. (2014) 85:799-805. doi: 10.1136/jnnp-2013-305505

27. Fallenius M, Raj R, Reinikainen M, Bendel S, Skrifvars MB. Association between high arterial oxygen tension and long-term survival after spontaneous intracerebral hemorrhage. Crit Care Med. (2016) 44:180-7. doi: 10.1097/CCM.0000000000001281

28. Lang M, Raj R, Skrifvars MB, Koivisto $T$, Lehto $H$, Kivisaari $\mathrm{R}$, et al. Early moderate hyperoxemia does not predict outcome after aneurysmal subarachnoid hemorrhage. Neurosurgery. (2016) 78:540-5. doi: 10.1227/NEU.0000000000001111

29. de Jonge E, Peelen L, Keijzers PJ, Joore H, de Lange D, van der Voort PH, et al. Association between administered oxygen, arterial partial oxygen pressure and mortality in mechanically ventilated intensive care unit patients. Crit Care. (2008) 12:R156. doi: 10.1186/cc7150

30. Seder DB, Riker RR, Jagoda A, Smith WS, Weingart SD. Emergency neurological life support: airway, ventilation, and sedation. Neurocrit Care. (2012) 17(Suppl. 1):S4-S20. doi: 10.1007/s12028-012-9753-6

31. Lodato RF. Decreased $\mathrm{O}_{2}$ consumption and cardiac output during normobaric hyperoxia in conscious dogs. J Appl Physiol (1985). (1989) 67:1551-9. doi: 10.1152/jappl.1989.67.4.1551

32. Floyd TF, Clark JM, Gelfand R, Detre JA, Ratcliffe S, Guvakov D, et al. Independent cerebral vasoconstrictive effects of hyperoxia and accompanying arterial hypocapnia at 1 ATA. J Appl Physiol (1985). (2003) 95:245361. doi: 10.1152/japplphysiol.00303.2003

33. Rousseau A, Tesselaar E, Henricson J, Sjoberg F. Prostaglandins and radical oxygen species are involved in microvascular effects of hyperoxia. J Vasc Res. (2010) 47:441-50. doi: 10.1159/000282667
34. Zaer H, Ketharanathan B, Carlsen JG, Shahbazi S, Sorensen JCH. Age as a prognostic factor in relation to surgical evacuation of spontaneous supratentorial intracerebral haemorrhage. Neurol Neurochir Pol. (2018) 52:750-5. doi: 10.1016/j.pjnns.2018.09.005

35. Lioutas VA, Marchina S, Caplan LR, Selim M, Tarsia J, Catanese L, et al. Endotracheal intubation and in-hospital mortality after intracerebral hemorrhage. Cerebrovasc Dis. (2018) 45:270-8. doi: 10.1159/000 489273

36. Boulanger M, Poon MT, Wild SH, Al-Shahi Salman R. Association between diabetes mellitus and the occurrence and outcome of intracerebral hemorrhage. Neurology. (2016) 87:870-8. doi: 10.1212/WNL.0000000000003031

37. Tao C, Hu X, Wang J, Ma J, Li H, You C. Admission neutrophil count and neutrophil to lymphocyte ratio predict 90 -day outcome in intracerebral hemorrhage. Biomark Med. (2017) 11:33-42. doi: 10.2217/bmm-201 6-0187

Conflict of Interest: The authors declare that the research was conducted in the absence of any commercial or financial relationships that could be construed as a potential conflict of interest.

Publisher's Note: All claims expressed in this article are solely those of the authors and do not necessarily represent those of their affiliated organizations, or those of the publisher, the editors and the reviewers. Any product that may be evaluated in this article, or claim that may be made by its manufacturer, is not guaranteed or endorsed by the publisher.

Copyright () $2021 \mathrm{Cai}, \mathrm{Ru}, \mathrm{Xu}, \mathrm{Wu}$, Gong, Yan and Shen. This is an open-access article distributed under the terms of the Creative Commons Attribution License (CC $B Y)$. The use, distribution or reproduction in other forums is permitted, provided the original author(s) and the copyright owner(s) are credited and that the original publication in this journal is cited, in accordance with accepted academic practice. No use, distribution or reproduction is permitted which does not comply with these terms. 